\title{
Structural Requirements for Modulating 4-Benzylpiperidine Carboxamides from Serotonin/Norepinephrine Reuptake Inhibitors to Triple Reuptake Inhibitors
}

\author{
Suresh Paudel ${ }^{1, \dagger}$, Eunae Kim ${ }^{2, \dagger}$, Anlin Zhu ${ }^{1, \dagger}$, Srijan Acharya ${ }^{1}$, Xiao Min ${ }^{1}$, Seung Hoon Cheon ${ }^{1}$ and \\ Kyeong-Man Kim ${ }^{1, *}$ \\ ${ }^{1}$ College of Pharmacy, Chonnam National University, Gwangju 61186, \\ ${ }^{2}$ College of Pharmacy, Chosun University, Gwangju 61452, Republic of Korea
}

\begin{abstract}
In this study, we determined the effect of 24 different synthetic 4-benzylpiperidine carboxamides on the reuptake of serotonin, norepinephrine, and dopamine (DA), and characterized their structure-activity relationship. The compounds with a two-carbon linker inhibited DA reuptake with much higher potency than those with a three-carbon linker. Among the aromatic ring substituents, biphenyl and diphenyl groups played a critical role in determining the selectivity of the 4-benzylpiperidine carboxamides toward the serotonin transporter (SERT) and dopamine transporter (DAT), respectively. Compounds with a 2-naphthyl ring were found to exhibit a higher degree of inhibition on the norepinephrine transporter (NET) and SERT than those with a 1-naphthyl ring. A docking simulation using a triple reuptake inhibitor $\mathbf{8 k}$ and a serotonin/norepinephrine reuptake inhibitor $\mathbf{7 j}$ showed that the regions spanning transmembrane domain (TM)1, TM3, and TM6 form the ligand binding pocket. The compound $\mathbf{8 k}$ bound tightly to the binding pocket of all three monoamine reuptake transporters; however, $7 \mathbf{j}$ showed poor docking with DAT. Co-expression of DAT with the dopamine $D_{2}$ receptor $\left(D_{2} R\right)$ significantly inhibited $D A$-induced endocytosis of $D_{2} R$ probably by reuptaking DA into the cells. Pretreatment of the cells with $\mathbf{8 f}$, which is one of the compounds with good inhibitory activity on DAT, blocked DAT-induced inhibition of $D_{2} R$ endocytosis. In summary, this study identified critical structural features contributing to the selectivity of a molecule for each of the monoamine transporters, critical residues on the compounds that bound to the transporters, and the functional role of a DA reuptake inhibitor in regulating $D_{2} R$ function.
\end{abstract}

Key Words: 4-Benzylpiperidine carboxamides, Serotonin reuptake inhibitor, Norepinephrine reuptake inhibitor, Triple reuptake inhibitor, Docking, Dopamine $\mathrm{D}_{2}$ receptor endocytosis

\section{INTRODUCTION}

Various neuropsychiatric health disorders, such as depression, anxiety, panic disorder, obsessive-compulsive disorder, and attention-deficit hyperactivity disorder, show overlap with respect to their pathogenic mechanisms and therapeutic regimens (Goodwin, 2015; Koyuncu et al., 2015). In addition to the selective serotonin reuptake inhibitors, such as fluoxetine (Jakubovski et al., 2016), serotonin/norepinephrine reuptake inhibitors (SNRIs), selective norepinephrine reuptake inhibitors, and norepinephrine/dopamine reuptake inhibitors are also conventionally used as antidepressants (Furukawa et al., 2016; Cipriani et al., 2018).
Currently used antidepressants exhibit a low remission rate and have several side effects (Trivedi, 2006; Culpepper et al., 2015), thereby demanding the development of new drugs/ treatment strategies. One of the approaches that is being used to tackle the aforementioned shortcomings is the development of multi-target drugs, which can be used for several diseases with a variety of symptoms and pathological mechanisms (Millan, 2006; Talevi, 2015). Multi-target drugs are known to possess improved therapeutic effects and have fewer side effects compared to single target drugs; they, thus, encourage improved patient compliance (Ramsay et al., 2018).

The monoamine hypothesis of depression states that the underlying pathophysiology of depression is based on the

\section{Open Access https://doi.org/10.4062/biomolther.2020.233}

This is an Open Access article distributed under the terms of the Creative Commons Attribution Non-Commercial License (http://creativecommons.org/licenses/by-nc/4.0/) which permits unrestricted non-commercial use, distribution, and reproduction in any medium, provided the original work is properly cited.
Received Dec 28, 2020 Revised Apr 1, 2021 Accepted Apr 2, 2021 Published Online May 31, 2021

\section{*Corresponding Author}

E-mail:kmkim@jnu.ac.kr

Tel: +82-62-530-2936, Fax: +82-62-530-2949

${ }^{\dagger}$ The first three authors contributed equally to this work. 
depletion of serotonin (5-HT), norepinephrine (NE), and/or dopamine (DA) in the central nervous system (Hirschfeld, 2000; Belmaker and Agam, 2008). However, current therapies solely target 5-HT and NE. They have a slow onset of action and other associated side effects (Prins et al., 2011), which may help explain the low remission rates. Thus, it is possible that these unsatisfactory therapeutic outcomes may be due to the negligence of dopamine's role in depression. For example, dopaminergic activity has been shown to be suppressed in patients with depression, and anhedonia has also been noted in most treatment-resistant patients of depression (Nestler and Carlezon, 2006; Sharma et al., 2015). Therefore, the possibility of using triple reuptake inhibitors (TRIs) as a treatment for depression is gaining increasing attention as they increase DA neurotransmission, which is not achieved by conventional treatments (Prins et al., 2011).

Previously, some arylalkanol-piperidine derivatives, which are structurally similar to 4-benzylpiperidine carboxamides, have been reported to exhibit triple reuptake inhibition and antidepressant effects (Zheng et al., 2012). In a previous study, we synthesized 24 compounds with a 4-benzylpiperidine carboxamide scaffold and analyzed their inhibitory activities on the serotonin transporter (SERT) and norepinephrine transporter (NET) (Paudel et al., 2015). However, in this study, the half maximal inhibitory concentration $\left(\mathrm{IC}_{50}\right)$ was determined for some and not all of the compounds and the effects of the 4-benzylpiperidine carboxamide compounds on the dopamine transporter (DAT) had not been tested. Thus, this study could not provide the necessary information for motivating a shift from SNRIs to TRIs for use as antidepressant drugs.

In the present study, we conducted reuptake inhibition assays of NET, SERT, and DAT using the synthetic 4-benzylpiperidine carboxamides (Paudel et al., 2015) and determined the structure-activity relationship (SAR) for these three transporters. In addition, docking simulation was conducted to predict how transporters interact with the synthesized compounds. Finally, we evaluated the effect of DAT inhibition on $D_{2} R$ function.

Our results revealed that the number of carbon linkers between $\mathrm{NH}$ and 4-benzylpiperidine and the nature of aromatic substituents play a critical role in determining the selectivity of 4-benzylpiperidine carboxamides to DAT and that the regulation of DAT activity determines dopamine receptor function.

\section{MATERIALS AND METHODS}

\section{Reagents}

GBR12909 dihydrochloride was purchased from Tocris Bioscience (Bristol, UK). Venlafaxine and dopamine were obtained from Sigma-Aldrich Chemical Co (St. Louis, MO, USA). $\left[{ }^{3} \mathrm{H}\right]-\mathrm{DA}$ and $\left[{ }^{3} \mathrm{H}\right]-5-\mathrm{HT}$ were purchased from PerkinElmer Life Sciences (Waltham, MA, USA). [ $\left.{ }^{3} \mathrm{H}\right]-S u l p i r i d e ~(75.7 \mathrm{Ci} / \mathrm{mmol})$ was purchased from PerkinElmer Life Sciences. The synthetic 4-benzylpiperidine carboxamides used in this study have been described in a previous study (Paudel et al., 2015). Human embryonic kidney (HEK)-293 cells were obtained from the American Type Culture Collection (Manassas, VA, USA) and maintained in minimal essential medium containing $10 \%$ fetal bovine serum, $100 \mathrm{U} / \mathrm{mL}$ penicillin, and $100 \mu \mathrm{g} / \mathrm{mL}$ streptomycin.

\section{Reuptake assay}

Reuptake assay was conducted using the HEK-293 cells stably expressing DAT, NET, and SERT. Cells were cultured in 24-well plates and washed with an uptake buffer comprising $5 \mathrm{mM}$ Tris base, $7.5 \mathrm{mM}$ HEPES, $120 \mathrm{mM} \mathrm{NaCl}, 5.4 \mathrm{mM} \mathrm{KCl}$, $1.2 \mathrm{mM} \mathrm{CaCl}_{2}, 1.2 \mathrm{mM} \mathrm{MgSO}_{4}, 1 \mathrm{mM}$ ascorbic acid, and $5 \mathrm{mM}$ glucose $(\mathrm{pH} 7.1)$. Thereafter, the cells were treated with the synthesized compounds at $37^{\circ} \mathrm{C}$ for $20 \mathrm{~min}$, followed by incubation with $\left[{ }^{3} \mathrm{H}\right]-\mathrm{DA}$ or $\left[{ }^{3} \mathrm{H}\right]-5-\mathrm{HT}$ (final concentration, $20 \mathrm{nM}$ ) for $5 \mathrm{~min}$. The cells were then washed thrice with ice cold uptake buffer and incubated overnight with phosphate-buffered saline containing $1 \%$ sodium dodecyl sulfate. Post incubation, samples were mixed with a scintillation cocktail and radioactivity was measured using the Wallac 1450 MicroBeta $^{\circledR}$ TriLux liquid scintillation counter (PerkinEImer). GBR12909 and venlafaxine were used as the reference compounds for the DA and NE/5-HT reuptake assay, respectively.

To determine the $\mathrm{IC}_{50}$ of the synthesized compounds, the concentration of the compounds was gradually increased and the percentage of inhibition was assessed for each concentration. GraphPad Prism 5 (GraphPad Software, San Diego, CA, USA) was used to construct the dose-response curve and determine the $\mathrm{IC}_{50}$.

\section{Docking simulation}

For the docking simulation, human SERT (hSERT), human NET (hNET), and human DAT (hDAT) were selected. The complex structure of hSERT bound to paroxetine was obtained from the Protein Data Bank (PDB ID: 5I6X) (Coleman et al., 2016). The 3-dimensional (3D) structures of DAT and NET are not currently available; thus, they were built by homology modeling. The sequences of hNET (UniProt ID: P23975) and hDAT (UniProt ID: Q01959) were obtained from UniProt database. Based on the X-ray structure of the Drosophila DAT (PDB ID: 4XP4) (Wang et al., 2015) with cocaine as the ligand for the transporter, the Swiss-Model (Waterhouse et al., 2018) server was used for modeling the protein structure. The sequence homology of hNET and hDAT was $58.9 \%$ and $55.0 \%$, and the QMEAN (Qualitative Model Energy ANalysis) values of the models were -3.49 and -3.42 , respectively, meaning that the model systems had medium quality and several partial regions were uncertain. The compound $\mathbf{7 j}$ and $\mathbf{8 k}$ were built by MarvinSketch software (ChemAxon, 2014) and optimized using AMBERff14SB force field (Maier et al., 2015). The visiualization of the binding interaction between the target transporter and the ligand was achieved using Chimera software (Pettersen et al., 2004). All docking simulation was performed using Autodock Vina (Trott and Olson, 2010).

\section{Endocytosis assay}

Endocytosis of dopamine $D_{2}$ receptor $\left(D_{2} R\right)$ was measured based on the hydrophilic properties of $\left[{ }^{3} \mathrm{H}\right]$-sulpiride (Kim et al., 2001). HEK-293 cells were transfected with $D_{2} R$, and after one day of transfection, the cells were seeded at a density of $1.5 \times 10^{5}$ cells/well in 24 -well plates. After $24 \mathrm{~h}$, the cells were stimulated with $10 \mu \mathrm{M}$ DA for 60 min and washed thrice with warm serum-free media. The cells were then incubated with $250 \mu \mathrm{L}$ of $\left[{ }^{3} \mathrm{H}\right]$-sulpiride (final concentration $2.2 \mathrm{nM}$ ) at $4^{\circ} \mathrm{C}$ for $150 \mathrm{~min}$ in the absence and presence of a competitive inhibitor $(10 \mu \mathrm{M}$ haloperidol). The cells were washed thrice with icecold serum-free media and then $1 \%$ sodium dodecyl sulfate was added. The samples were mixed with $2 \mathrm{~mL}$ Lefko-Fluor 
Table 1. List of the half-maximal inhibitory concentrations $\left(\mathrm{IC}_{50}\right)$ of 4-benzylpiperidine carboxamides for SERT, NET, and DAT

\begin{tabular}{|c|c|c|c|c|c|}
\hline No & $\mathrm{n}$ & $\mathrm{R}^{1}$ & $5-\mathrm{HT}$ & $\mathrm{NE}$ & DA \\
\hline $7 a$ & 3 & 3,5-dichlorophenyl & $0.19-0.84$ & $0.12-0.56$ & $>10$ \\
\hline $7 b$ & 3 & 2-indolyl & $0.24-0.92$ & $0.12-0.56$ & $>10$ \\
\hline 7c & 3 & benzo(b)furanyl & $0.45-0.54$ & $0.54-0.71$ & $>10$ \\
\hline $7 d$ & 3 & benzo $(b)$ thiophenyl & $0.32-0.61$ & $0.98-10$ & $>10$ \\
\hline $7 e$ & 3 & 4-biphenyl & $0.04-0.07$ & $0.01-2.12$ & $>10$ \\
\hline $7 f$ & 3 & diphenylacetyl & $>10$ & $0.83-3.38$ & $0.09-0.40$ \\
\hline $7 \mathrm{~g}$ & 3 & bis(4-chlorophenyl)acetyl & $>10$ & $1.13-3.62$ & $0.78-2.34$ \\
\hline $7 \mathrm{~h}$ & 3 & 2-quinolyl & $0.03-50$ & $1.02-5.66$ & $>10$ \\
\hline $7 i$ & 3 & 1-naphthyl & $7.51^{\star}$ & $0.87-7.43$ & $8.09^{*}$ \\
\hline $7 \mathrm{j}$ & 3 & 2-naphthyl & $0.06-0.18$ & $0.15-0.76$ & $>10$ \\
\hline $7 \mathrm{k}$ & 3 & 6-bromo-2-naphthyl & $0.31-46$ & $0.53-1.45$ & $>10$ \\
\hline 71 & 3 & 6-methoxy-2-naphthyl & $0.23-0.45$ & $0.02-2.72$ & $>10$ \\
\hline $8 a$ & 2 & 3,5-dichlorophenyl & $0.55-6.22$ & $0.55-1.88$ & $0.37-1.17$ \\
\hline $8 b$ & 2 & 2-indolyl & $0.15-1.12$ & $0.20-3.82$ & $0.34-1.68$ \\
\hline $8 c$ & 2 & benzo(b)furanyl & $0.19-2.79$ & $1.52-2.20$ & $0.96-2.59$ \\
\hline $8 d$ & 2 & benzo $(b)$ thiophenyl & $0.18-5.39$ & $0.60-1.26$ & $0.84-2.13$ \\
\hline $8 e$ & 2 & 4-biphenyl & $0.986^{*}$ & $0.17-1.28$ & $>10$ \\
\hline $8 f$ & 2 & diphenylacetyl & $>10$ & $0.29-16$ & $0.32-3.5$ \\
\hline $8 g$ & 2 & bis(4-chlorophenyl)acetyl & $>10$ & $>10$ & $0.195-2.05$ \\
\hline $8 \mathrm{~h}$ & 2 & 2-quinolyl & $0.42-1.45$ & $0.53-1.45$ & $>10$ \\
\hline $8 \mathrm{i}$ & 2 & 1-naphthyl & $>10$ & $0.18-105$ & $5.37^{*}$ \\
\hline 8j & 2 & 2-naphthyl & $0.39-1.31$ & $0.28-0.57$ & $0.71-3.70$ \\
\hline $8 \mathrm{k}$ & 2 & 6-bromo-2-naphthyl & $0.48-0.83$ & $0.02-0.19$ & $0.41-1.73$ \\
\hline 81 & 2 & 6-methoxy-2-naphthyl & $0.49-3.19$ & $0.20-0.47$ & $1.08-8.40$ \\
\hline- & - & Venlafaxine & $0.07-0.52$ & $2.16-3.01$ & - \\
\hline- & - & GBR12909 & - & - & $0.02-0.08$ \\
\hline
\end{tabular}

The $\mathrm{IC}_{50}$ are shown as $95 \%$ confidence intervals. Unit of the concentration is $\mu \mathrm{M}$.

${ }^{*} 95 \%$ confidence interval could not be determined because the range was too wide.

scintillation fluid and counted on a liquid scintillation analyzer (1450 MicroBeta Trilux, PerkinEImer).

\section{Statistical analysis}

Values are expressed as the mean \pm standard deviation. Statistical significance of the data was analyzed using a oneway analysis of variance with Tukey's post hoc test using GraphPad Prism 5 (GraphPad Software). A p-value $<0.05$ was considered significant.

\section{RESULTS}

\section{The number of carbons in the linker region is critical in determining TRI activity}

As shown in Table 1, most of the 4-benzylpiperidine carboxamides exhibited similar degrees of inhibition toward NET and SERT, irrespective of the number of carbons in the linker region between $\mathrm{NH}$ and 4-benzylpiperidine (n). Interestingly, compounds containing two carbons in the linker region had a stronger inhibitory effect on DAT than those with three carbons in the linker region. Thus, these results suggest that the number of carbons in the linker region is critical in converting compounds that are SNRIs into TRIs.

\section{Effect of $\mathbf{R}^{1}$ substituents on SERT inhibition}

As shown in Table 1, 4-benzylpiperidine carboxamides exhibited varying degrees of inhibition depending on the aromatic substituent present at $\mathrm{R}^{1}$. In contrast to the biphenyl substituted compound (e) that exhibited a strong inhibition of SERT, compounds with diphenyl substitution ( $\mathbf{f}, \mathbf{g})$ exhibited very weak inhibition of SERT, irrespective of the number of carbons in the linker region. Compounds with fused heterocyclic substitutions on $\mathrm{R}^{1}$ exerted similar degrees of inhibition on SERT, regardless of the number of carbons in the linker region (b, c, d). For naphthyl derivatives, compounds with a 2-naphthyl substitution ( $\mathrm{j}-\mathrm{I})$ at $\mathrm{R}^{1}$ exhibited a higher degree of inhibition than those with a 1-naphthyl substitution (i), regardless of the $n$ value. Inhibition of SERT by substituted 2-naphthyl derivative compounds (8k and $\mathbf{8} \mathbf{I}$ ) was greater than that by unsubstituted 2-naphthyl derivative $8 \mathbf{j}$. SERT inhibition by compounds containing a naphthyl ring was in the following order: 6-bromo $(\mathbf{8 k})<6$-methoxy $(\mathbf{8 I})<2$-naphthyl $(\mathbf{8 j})$. The SAR of the 4-benzylpiperidine carboxamides with SERT is summarized in Fig. 1.

\section{Effects of $\mathbf{R}^{1}$ substituents on NET inhibition}

Most of the 4-benzylpiperidine carboxamides exhibited a higher degree of inhibition of NET than the control drug, ven- 
$\mathrm{R} 1=$
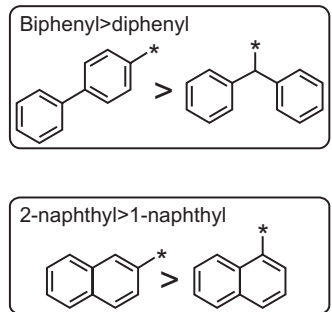

Fig. 1. Structure-activity relationship of 4-benzylpiperidine carboxamides for the inhibition of serotonin (5-HT) reuptake. Compounds with a biphenyl ring exhibit a higher degree of inhibition of the serotonin transporter than those with a diphenyl ring. Compounds with a 2-naphthyl substitution generally exhibit enhanced inhibition of 5-HT reuptake than those with a 1-naphthyl substitution.

lafaxine. In eight out of twelve compounds, compounds with three carbons in the linker region exhibited a stronger inhibition of NET compared to those with two carbons in the linker region. As for SERT, the compound with a biphenyl substitution (e) exhibited a stronger inhibition of NET compared to those with diphenyl substituents (f, g). For the compounds with a naphthyl substitution, 2-naphthyl derivatives (j-I) exhibited a higher degree of inhibition than those with a 1-naphthyl substitution (i). The SAR of the 4-benzylpiperidine carboxamides with NET is summarized in Fig. 2.

\section{Effects of $\mathbf{R}^{1}$ substituents on DAT inhibition}

In contrast to SERT and NET inhibition, all 4-benzylpiperidine carboxamides with two carbons in the linker region exhibited a higher degree of inhibition of DAT compared to those with three carbons in the linker region. Interestingly, DA reuptake inhibition of a compound was highly correlated with the presence of a diphenyl acetyl substitution (f, g) irrespective of the number of carbons in the linker region. Conversely, the compound with a biphenyl substitution (e), which exhibited a stronger inhibition of SERT and NET, showed a very weak inhibition of DAT, irrespective of the number of carbons in the linker region. The effect of the compounds with fused heterocyclic ring (b, c, d) on DAT inhibition was not very clear. As for NET, compounds with a 2-naphthyl substitution (8j-8I) exhibited a higher degree of inhibition than those with a 1-naphthyl substitution (8i). The SAR of the 4-benzylpiperidine carboxamides with DAT is summarized in Fig. 3.

\section{Docking study}

To predict the binding conformation of the compounds with monoamine transporters, molecular docking studies were conducted. For this, the most potent compounds $\mathbf{8 k}$ and $\mathbf{7 j}$, whose inhibitory activity greatly varies according to a change in the number of carbon atoms in the linker region were selected for the docking simulation. As shown in Fig. 4, 8k bound to all three transporters while $7 \mathbf{j}$ bound only to hSERT and hNET. In accordance with the reuptake results, $7 \mathrm{j}$ poorly bound to the open-site near the extracellular region of hDAT (data was not shown).

As shown in Fig. 4A, 8k and $7 \mathbf{j}$ fully occupied the region from the central binding site to the open-site into the extracellular space in hSERT. Although the residues in contact with $\mathbf{8 k}$ and $7 \mathbf{j}$ were similar, the binding pose of the two compounds
$\mathrm{R} 1=$
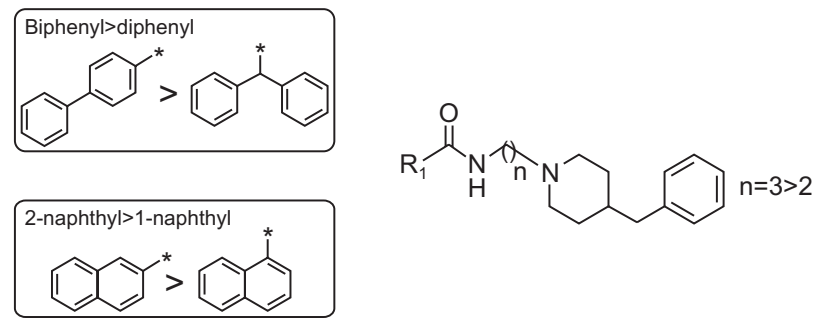

Fig. 2. Structure-activity relationship of 4-benzylpiperidine carboxamides for the inhibition of norepinephrine reuptake. Compounds with a biphenyl ring exhibit a higher degree of inhibition of the norepinephrine transporter than those with a diphenyl ring; particularly, those with bis(4-chlorophenyl)acetyl substitution. Compounds with a 2-naphthyl substitution exhibit a higher degree of inhibition than those with a 1-naphthyl substitution regardless of the number of carbons in the linker region.

$\mathrm{R} 1=$
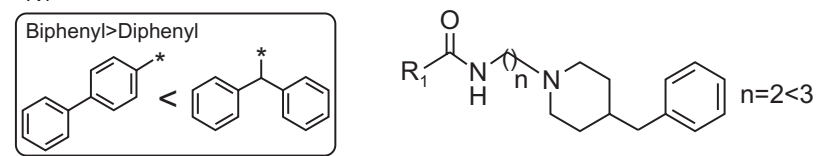

Fig. 3. Structure-activity relationship of 4-benzylpiperidine carboxamides for the inhibition of dopamine (DA) reuptake. Compounds with two carbons in the linker region exhibit a higher degree of inhibition of the dopamine transporter compared to those with three carbons in the linker region. Additionally, compounds with a diphenyl group exhibit stronger inhibition of DA reuptake compared to those with a biphenyl group.

was different. The 4-benzylpiperidine moiety of $\mathbf{8 k}$ tightly fit in the central binding site of the cell membrane-spanning region where it showed hydrophobic interactions with Ala169, Tyr175, and lle172 in transmembrane (TM)3 and with Phe341 in TM6. The 6-bromo-2-naphthyl substituent of $\mathbf{8 k}$ stacked in parallel with Phe335 in TM6 and was surrounded by Ala331 in TM6 and Phe556 in TM11. These observations are consistent with the binding properties of the compound $\mathbf{2 q}$ that had been found to show potent inhibition in a previous study (Paudel et al., 2017b). In contrast, the 2-naphthyl substituent of $7 \mathbf{j}$ was located at the central binding site and the 4-benzylpiperidine moiety of $7 \mathbf{j}$ blocked the outward-open site into the extracellular space. In spite of the different binding pose between the two compounds, they were flexible enough to form the same 3D shape. Notably, the existence of bromine determined the orientation of the ligand, that is, the 6-bromo-2-naphthyl substituent was enough big to fit into the central binding pocket.

As shown in Fig. $4 \mathrm{~B}$, the best binding pose of $\mathbf{8 k}$ and $7 \mathbf{j}$ was similar for hNET. The 4-benzylpiperidine moiety faced the inner pocket of hNET and had multiple interactions with the hydrophobic sidechains, for example, Phe72/Ala77 in TM1, Val148/Tyr152 in TM3, and Phe323 in TM6. In addition, the 2-naphthyl substituent showed a strong $\pi$ - $\pi$ stacking interaction with Phe317 in TM6.

In Fig. 4C, the possible binding pose of $\mathbf{8 k}$ in hDAT was similar to that with hNET. The aromatic ring on both sides was surrounded with the following hydrophobic residues; Phe76/ Ala81 in TM1, Val152/Tyr156 in TM3, and Phe320/Phe326 in TM6. When the three complexes between $\mathbf{8 k}$ and the trans- 

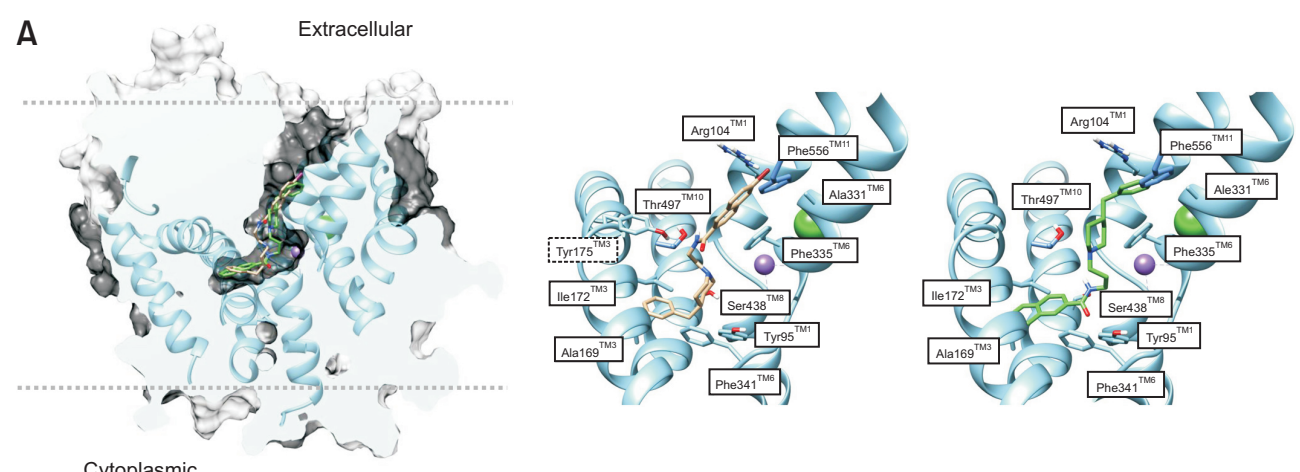

Cytoplasmic
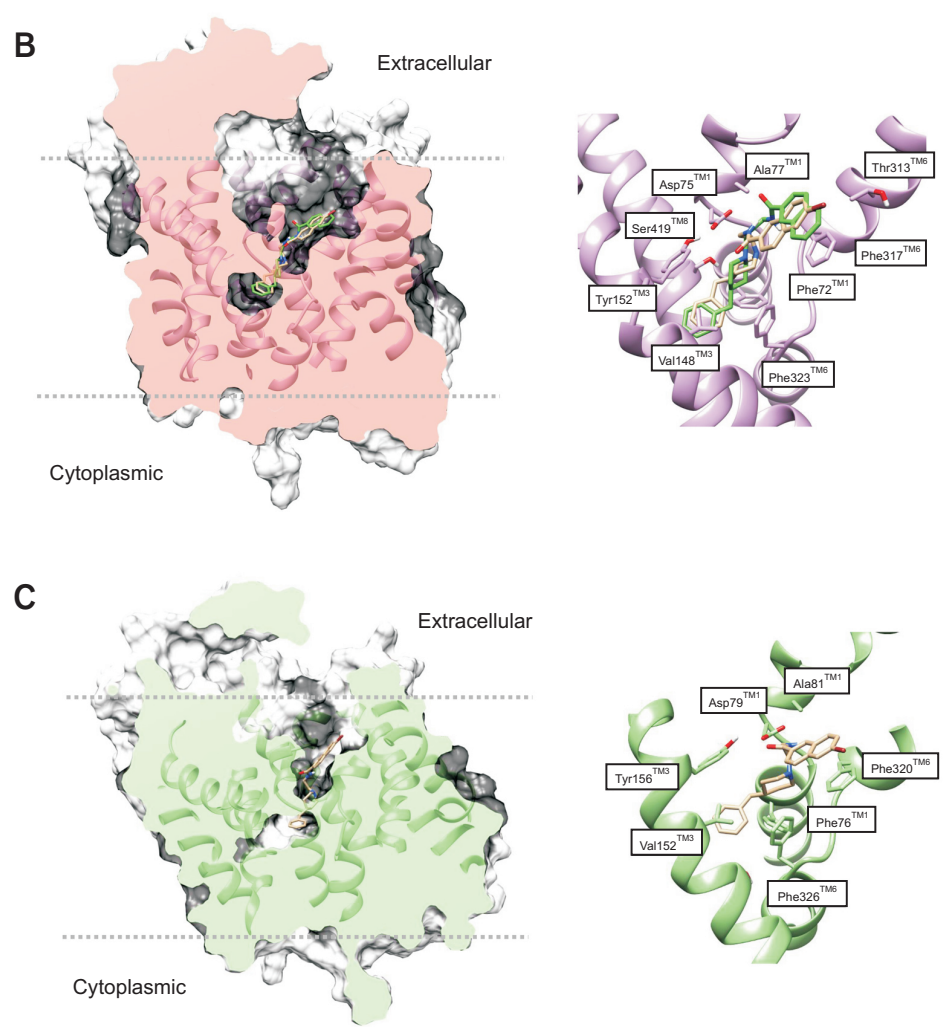

Fig. 4. The predicted binding pose of $\mathbf{8 k}$ (stick, tan) and $\mathbf{7 j}$ (stick, green) for human serotonin transporter (A), human norepinephrine transporter (B), and human dopamine transporter $(C)$. The purple colored sphere designates sodium $\left(\mathrm{Na}^{+}\right)$and the green colored sphere designates chloride $\left(\mathrm{Cl}^{-}\right)$. The compound $\mathbf{8 k}$ binds to all three monoamine reuptake transporters.

porters were aligned, the important hydrophobic residues in the binding pocket were found to be conserved-Tyr95 of hSERT, Phe72 of hNET, and Phe76 of hDAT in TM1; lle172 of SERT, Val148 of hNET, and Val152 of hDAT in TM3; Phe335/ Phe341 of hSERT, Phe317/Phe323 of hNET, and Phe320/ Phe326 of hDAT in TM6. Three TMs, TM1, TM3, and TM6, formed the essential portion of the binding pocket, and the compound $\mathbf{8 k}$-which fit well into the pocket-inhibited the reuptake of all monoamines. The poor docking between $7 \mathbf{j}$ and hDAT supports the results obtained from functional assay. Further studies are needed to identify specific residues that determines the selectivity of hDAT for the target.

\section{Functional studies of dopamine reuptake inhibition}

It is known that reuptake transporters regulate the synaptic concentration of neurotransmitters. Reuptake of the released neurotransmitters into presynaptic nerve terminals is responsible for the rapid termination of neurotransmission in the synapses (Masson et al., 1999; Torres et al., 2001). To understand the inhibition of monoamine reuptake functionally, we determined the effect of 4-benzylpiperidine carboxamides on the endocytosis of dopamine $D_{2}$ receptor $\left(D_{2} R\right)$.

Agonist stimulation of $G$ protein-coupled receptors (GP$\mathrm{CRs}$ ) promotes the exchange of guanosine diphosphate (GDP)/guanosine triphosphate (GTP) at the nucleotide-binding pocket of the $\mathrm{G} \alpha$ subunit. GTP binding induces conformational changes in the $\mathrm{G} \alpha$ subunit, resulting in the dissociation of $G \alpha$ from the $G \beta \gamma$ subunit and from the receptor (Hepler and Gilman, 1992). After agonist stimulation, the receptor is phosphorylated by GPCR kinases, enhancing the binding of 


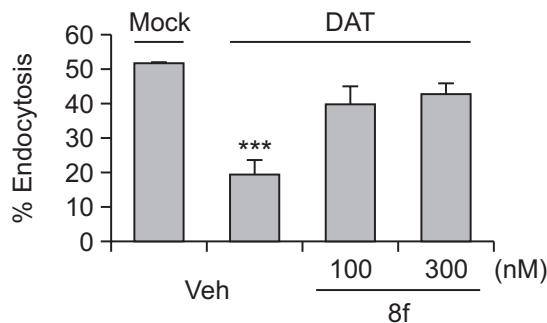

Fig. 5. Effects of the dopamine transporter (DAT) and its inhibitor $\mathbf{8 f}$ on the dopamine-induced endocytosis of dopamine $D_{2}$ receptor. Human embryonic kidney-293 cells were transfected with $1 \mu \mathrm{g}$ of dopamine $D_{2}$ receptor $\left(D_{2} R\right)$ in pCMV5 with or without $3 \mu \mathrm{g}$ of DAT. Cells were pretreated with vehicle (Veh), $100 \mathrm{nM}$ or $300 \mathrm{nM} 8 \mathrm{f}$ for $20 \mathrm{~min}$, followed by $10 \mu \mathrm{M}$ dopamine for $1 \mathrm{~h}$. ${ }^{* * *} p<0.001$ compared to other groups $(n=3)$.

$\beta$-arrestins, which connect to adaptors, such as adaptor protein (AP)-2 and clathrin, leading to the endocytosis of GPCRs (Zhang and Kim, 2017).

$D_{2} R$ also undergoes endocytosis in response to agonist stimulation (Kim et al., 2001), and the functional role of $D_{2} R$ endocytosis was proposed to be the resensitization of desensitized receptors (Cho et al., 2010). As shown in Fig. 5, treatment of the cells expressing $D_{2} R$ evoked approximately $40 \%$ of receptor endocytosis, and co-expression of DAT significantly inhibited this endocytosis of $D_{2} R$. Pretreatment with 8f, which inhibits DAT, restored the DAT-mediated inhibition of $D_{2} R$ endocytosis almost up to the basal level. These results suggest that 4-benzylpiperidine carboxamides can have profound influences on the signaling of monoamine neurotransmitters, for example, dopamine signaling via $D_{2} R$.

\section{DISCUSSION}

In the current study, we determined the $\mathrm{IC}_{50}$ of all $24 \mathrm{com}$ pounds for the three monoamine transporters, and analyzed the SAR that helped determine the selectivity of these compounds for the three transporters. Here we demonstrated two principles that can be applied to derive TRI compounds from SNRIs. First, the number of carbons in the linker region connecting $\mathrm{NH}$ and 4-benzylpiperidine determines the selectivity toward DAT, and switching three carbons to two carbons can modify SNRIs into TRIs. Second, the presence of biphenyl and diphenyl groups at the aromatic substitution position determines the selectivity for SERT/NET and DAT, respectively. Since DA is structurally more similar to NE than it is to $5-\mathrm{HT}$, it is generally more difficult to achieve high differential selectivity between DAT and NET (Paudel et al., 2017a). Thus, it is interesting that this study proposed principles that can be applied for achieving high differential selectivity between DAT and NET/SERT rather than between SERT and NET/DAT.

DAT and its inhibitors regulate the endocytosis of $D_{2} R$ and in turn impact synaptic transmission in the dopaminergic system. DAT lowers the concentration of DA in the synaptic cleft and thus, is expected to have multiple effects on the signaling or regulatory processes of dopaminergic neurons. DAT inhibitors, by reversing the reuptake by DAT, are expected to trigger various pathophysiological states, including drug addiction and psychological symptoms. Even though we did not test for this in noradrenergic and serotonergic neurons, similar regulatory outcomes are expected for their synaptic transmissions.

As expected from the reuptake assay, docking simulation showed that the TRI $\mathbf{8 k}$ fit into the binding pockets of all of the three transporters; however, the SNRI 7j was able to bind to NET and SERT. It was difficult to elucidate the molecular basis behind the poor docking of $7 \mathbf{j}$ with hDAT, partly because of a certain degree of uncertainty in the docking model.

In conclusion, the combination of the number of carbon linkers between $\mathrm{NH}$ and 4-benzylpiperidine and the nature of the aromatic substituents in the compounds contribute to the selectivity of 4-benzylpiperidine carboxamides for monoamine reuptake transporters. We believe that our findings will be useful for the further design and development of novel SNRIs and TRIs.

\section{CONFLICT OF INTEREST}

The authors declare no financial or commercial conflict of interest.

\section{ACKNOWLEDGMENTS}

This study was financially supported by Chonnam National University (Grant number: 2020-1874).

\section{REFERENCES}

Belmaker, R. H. and Agam, G. (2008) Major depressive disorder. N. Engl. J. Med. 358, 55-68.

ChemAxon (2014) MarvinSketch (version 6.2.2). Calculation module developed by ChemAxon. Available from: http://www.chemaxon. com/products/marvin/marvinsketch/.

Cho, D., Zheng, M., Min, C., Ma, L., Kurose, H., Park, J. H. and Kim, K. M. (2010) Agonist-induced endocytosis and receptor phosphorylation mediate resensitization of dopamine $\mathrm{D}(2)$ receptors. Mol. Endocrinol. 24, 574-86.

Cipriani, A., Furukawa, T. A., Salanti, G., Chaimani, A., Atkinson, L. Z., Ogawa, Y., Leucht, S., Ruhe, H. G., Turner, E. H., Higgins, J. P. T., Egger, M., Takeshima, N., Hayasaka, Y., Imai, H., Shinohara, K., Tajika, A., loannidis, J. P. A. and Geddes, J. R. (2018) Comparative efficacy and acceptability of 21 antidepressant drugs for the acute treatment of adults with major depressive disorder: a systematic review and network meta-analysis. Lancet 391, 1357-1366.

Coleman, J. A., Green, E. M. and Gouaux, E. (2016) X-ray structures and mechanism of the human serotonin transporter. Nature $\mathbf{5 3 2}$, 334-339.

Culpepper, L., Muskin, P. R. and Stahl, S. M. (2015) Major depressive disorder: understanding the significance of residual symptoms and balancing efficacy with tolerability. Am. J. Med. 128, S1-S15.

Furukawa, T. A., Salanti, G., Atkinson, L. Z., Leucht, S., Ruhe, H. G., Turner, E. H., Chaimani, A., Ogawa, Y., Takeshima, N., Hayasaka, Y., Imai, H., Shinohara, K., Suganuma, A., Watanabe, N., Stockton, S., Geddes, J. R. and Cipriani, A. (2016) Comparative efficacy and acceptability of first-generation and second-generation antidepressants in the acute treatment of major depression: protocol for a network meta-analysis. BMJ Open 6, e010919.

Goodwin, G. M. (2015) The overlap between anxiety, depression, and obsessive-compulsive disorder. Dialogues Clin. Neurosci. 17, 24960.

Hepler, J. R. and Gilman, A. G. (1992) G proteins. Trends Biochem. Sci. 17, 383-387.

Hirschfeld, R. M. (2000) History and evolution of the monoamine hypothesis of depression. J. Clin. Psychiatry 61 Suppl 6, 4-6. 
Jakubovski, E., Varigonda, A. L., Freemantle, N., Taylor, M. J. and Bloch, M. H. (2016) Systematic review and meta-analysis: doseresponse relationship of selective serotonin reuptake inhibitors in major depressive disorder. Am. J. Psychiatry 173, 174-183.

Kim, K. M., Valenzano, K. J., Robinson, S. R., Yao, W. D., Barak, L. S. and Caron, M. G. (2001) Differential regulation of the dopamine D2 and D3 receptors by $\mathrm{G}$ protein-coupled receptor kinases and betaarrestins. J. Biol. Chem. 276, 37409-37414

Koyuncu, A., Ertekin, E., Yuksel, C., Aslantas Ertekin, B., Celebi, F., Binbay, Z. and Tukel, R. (2015) Predominantly inattentive type of ADHD is associated with social anxiety disorder. J. Atten. Disord. 19, 856-864.

Maier, J. A., Martinez, C., Kasavajhala, K., Wickstrom, L., Hauser, K. E. and Simmerling, C. (2015) ff14SB: improving the accuracy of protein side chain and backbone parameters from ff99SB. J. Chem. Theory Comput. 11, 3696-3713.

Masson, J., Sagne, C., Hamon, M. and El Mestikawy, S. (1999) Neurotransmitter transporters in the central nervous system. Pharmacol. Rev. 51, 439-464.

Millan, M. J. (2006) Multi-target strategies for the improved treatment of depressive states: conceptual foundations and neuronal substrates, drug discovery and therapeutic application. Pharmacol. Ther. 110, 135-370.

Nestler, E. J. and Carlezon, W. A., Jr. (2006) The mesolimbic dopamine reward circuit in depression. Biol. Psychiatry 59, 1151-1159.

Paudel, S., Acharya, S., Yoon, G., Kim, K. M. and Cheon, S. H. (2017a) Design, synthesis and in vitro activity of 1,4-disubstituted piperazines and piperidines as triple reuptake inhibitors. Bioorg. Med. Chem. 25, 2266-2276.

Paudel, S., Cao, Y., Guo, S., An, B., Kim, K. M. and Cheon, S. H. (2015) Design and synthesis of 4-benzylpiperidine carboxamides as dual serotonin and norepinephrine reuptake inhibitors. Bioorg. Med. Chem. 23, 6418-6426.

Paudel, S., Min, X., Acharya, S., Khadka, D. B., Yoon, G., Kim, K. M. and Cheon, S. H. (2017b) Triple reuptake inhibitors: design, synthesis and structure-activity relationship of benzylpiperidinetetrazoles. Bioorg. Med. Chem. 25, 5278-5289.

Pettersen, E. F., Goddard, T. D., Huang, C. C., Couch, G. S., Green- blatt, D. M., Meng, E. C. and Ferrin, T. E. (2004) UCSF Chimera--a visualization system for exploratory research and analysis. J. Comput. Chem. 25, 1605-1612.

Prins, J., Olivier, B. and Korte, S. M. (2011) Triple reuptake inhibitors for treating subtypes of major depressive disorder: the monoamine hypothesis revisited. Expert Opin. Investig. Drugs 20, 1107-1130.

Ramsay, R. R., Popovic-Nikolic, M. R., Nikolic, K., Uliassi, E. and Bolognesi, M. L. (2018) A perspective on multi-target drug discovery and design for complex diseases. Clin. Transl. Med. 7, 3.

Sharma, H., Santra, S. and Dutta, A. (2015) Triple reuptake inhibitors as potential next-generation antidepressants: a new hope? Future Med. Chem. 7, 2385-2406.

Talevi, A. (2015) Multi-target pharmacology: possibilities and limitations of the "skeleton key approach" from a medicinal chemist perspective. Front. Pharmacol. 6, 205.

Torres, G. E., Yao, W. D., Mohn, A. R., Quan, H., Kim, K. M., Levey, A. I., Staudinger, J. and Caron, M. G. (2001) Functional interaction between monoamine plasma membrane transporters and the synaptic PDZ domain-containing protein PICK1. Neuron 30, 121-134.

Trivedi, M. H. (2006) Major depressive disorder: remission of associated symptoms. J. Clin. Psychiatry 67 Suppl 6, 27-32.

Trott, O. and Olson, A. J. (2010) AutoDock Vina: improving the speed and accuracy of docking with a new scoring function, efficient optimization, and multithreading. J. Comput. Chem. 31, 455-461.

Wang, K. H., Penmatsa, A. and Gouaux, E. (2015) Neurotransmitter and psychostimulant recognition by the dopamine transporter. Nature 521, 322-327.

Waterhouse, A., Bertoni, M., Bienert, S., Studer, G., Tauriello, G., Gumienny, R., Heer, F. T., de Beer, T. A. P., Rempfer, C., Bordoli, L., Lepore, R. and Schwede, T. (2018) SWISS-MODEL: homology modelling of protein structures and complexes. Nucleic Acids Res. 46, W296-W303.

Zhang, X. and Kim, K. M. (2017) Multifactorial regulation of G proteincoupled receptor endocytosis. Biomol. Ther. (Seoul) 25, 26-43.

Zheng, Y. Y., Guo, L., Zhen, X. C. and Li, J. Q. (2012) Synthesis and antidepressant activity of arylalkanol-piperidine derivatives as triple reuptake inhibitors. Eur. J. Med. Chem. 54, 123-136. 